\title{
Villes-frontières et réseaux informels sahéliens (Gaya-Malanville-Kamba)
}

\section{Olivier Walther, Luxembourg}

\section{Sahel: une conception mobile de l'espace}

En nommant Sahel ce «rivage» atteint au sortir de l'aridité saharienne, les marchands arabes précoloniaux ont contribué à alimenter deux discours géographiques contradictoires en ce qui concerne l'Afrique sahélienne. S'appuyant sur une vision déterministe des rapports entre l'environnement et les sociétés, le premier discours interprète le «rivage sahélien» comme une ligne de front délimitant une zone aux attributs bioclimatiques intermédiaires entre les domaines sahariens et soudanais, à laquelle correspond également un «genre de vie» particulier. Constatant les insuffisances de cette vision, notamment l'impossibilité de s'appuyer sur des moyennes de précipitations sans cesse irrégulières pour en déduire des comportements humains, le second discours s'attache à montrer que le «rivage sahélien» peut être conçu dans une perspective dynamique au sein de laquelle la «formule de contact» sahélienne (RETAILlÉ 1989) est soumise aux flux méridiens des populations agricultrices et pastorales. Récusant la représentation du «front» comme ligne d'affrontement entre deux formes concurrentes d'organisation de l'espace, certains géographes imaginent alors le Sahel comme un espace dans lequel la lutte que les hommes mènent contre l'incertitude climatique rend nécessaire d'accorder une importance déterminante à la circulation des biens et des personnes. Dans la mesure où les échanges empruntent des canaux structurés par des villes de taille et de fonctions différentes, mais dont la principale caractéristique est de s'ordonner selon des canaux non hiérarchisés, cette forme d'organisation territoriale s'apparente à une conception mobile de l'espace (Retaillé 2005).

C'est ce qui explique sans doute que l'essentiel des activités économiques sahéliennes se localise aujourd'hui dans ce qui est appelé le secteur informel au point de vue sectoriel alors que, du point de vue territorial, ces activités trouvent un terreau fécond dans les zones frontalières. Au travers de nouvelles limites, la colonisation et l'Etat-nation ont en effet reconstitué de nouvelles lignes d'échanges, sur lesquelles sont venues s'implanter des activités économiques liées aux différentiels monétaires et législatifs et organisées en réseaux informels. Ces activités, contrôlées par des acteurs que le langage populaire appelle patrons, sont favorisées par l'urbanisation extrêmement rapide que connaît le sous-continent (WaLther 2004). Malgré leur importance stratégique, les petites et moyennes villes situées sur les frontières n'ont pourtant pas fait jusqu'ici l'objet de programmes spécifiques, les politiques relatives aux centres urbains traitant de l'échelle locale ou nationale, en contradiction avec les stratégies régionales ou supranationales des acteurs économiques (Satterthwaite \& Tacoli 2003). Or, les marchands les plus influents ignorent à la fois l'Etat et les organismes de régulation et établissent des relations d'affaires qui s'étendent à une échelle internationale à partir des zones frontalières.

\section{Un régime de spatialité spécifique aux villes- frontières?}

Dans ce contexte, l'objectif de l'article est de comprendre le mode d'organisation économique spécifique aux petits centres urbains qui composent les espaces frontaliers sahéliens, en s'interrogeant sur leur concurrence ou leur complémentarité éventuelle à l'intérieur d'un régime de spatialité particulier. En s'appuyant sur l'exemple du carrefour économique de GayaMalanville-Kamba situé à la frontière entre le Niger, le Bénin et le Nigeria, il questionne le rôle de la villefrontière ainsi que le jeu des acteurs marchands localement dominants. L'analyse géographique de ce carrefour frontalier dont les potentialités résultent d'une combinaison de conditions favorables au développement d'une agriculture de rente et d'éléments stratégiques liés à sa situation à la charnière sahélienne, permet alors de montrer l'importance des logiques locales dans les modèles usuellement élaborés par les bailleurs de fonds internationaux.

Ce faisant, l'article se fonde sur l'hypothèse que les relations entretenues par les marchés des villes-frontières sont fondées sur une centralité mobile et que, par conséquent, les politiques de développement fondées sur des conceptions fonctionnalistes de l'activité économique ne permettent pas d'identifier les logiques circulatoires qui structurent les territoires sahéliens. Soutenus par une vision productiviste de l'espace économique et par la nécessité d'y prévenir les famines par l'accroissement de la production locale, les programmes actuels des agences de développement et des Etats sahéliens délaissent singulièrement les villes-marchés des zones frontalières et les réseaux informels, qui constituent pourtant des milieux où se sont reconstituées des logiques économiques adaptées à la gestion de l'incertitude.

La contradiction qui résulte des logiques étatiques, appuyées par les programmes de développement et 
les acteurs économiques locaux reconnus comme des patrons conduit à une forte désarticulation des espaces sahéliens, aujourd'hui considérés comme des lieux marginaux et enclavés. L'étude des potentialités du carrefour de Gaya-Malanville-Kamba postule au contraire que la marginalité et l'enclavement présumés du Sahel résultent d'une conception importée dans laquelle l'aspect circulatoire est négligé, ce qui ne permet pas de rendre adéquatement de l'adaptabilité des espaces sahéliens. En effet, confrontées à la nécessité de lutter contre l'incertitude climatique, les sociétés sahéliennes n'ont eu de cesse de vouloir préserver la souplesse de leurs réseaux sociaux et économiques. Ce faisant, elles ont engendré une dynamique permettant une gestion originale de l'aléatoire, dans laquelle l'accent est mis sur la diversification des pratiques économiques associées à une extrême mobilité.

\section{Méthodes}

Les méthodes utilisées dans cette étude associent des enquêtes empiriques effectuées entre 2003 et 2006 en partenariat avec l'Agence Nigérienne pour la Promotion de l'Irrigation Privée (ANPIP), à des éléments interprétatifs et modélisateurs (JAUBERT et al. 2005). Au plan quantitatif, les statistiques du Ministère du Développement Communautaire et de la Direction générale des Douanes du Niger ont servi de base à l'analyse des flux frontaliers. Elles ont été complétées par une enquête menée sur 47 sites de production situés dans des environnements à fort potentiel productif dans les départements de Gaya (Niger), de l'Alibori (Bénin) et l'Etat de Kebbi (Nigeria), ainsi que par des entretiens menés auprès de soixante détaillants des trois marchés concernés et de quinze grands commerçants actifs dans l'import-export.

\section{Les villes-marchés et la frontière dans la théorie géographique}

Longtemps cantonnées à la critique de l'artificialité des limites coloniales, les contributions des géographes à la théorie des frontières sahéliennes s'attachent aujourd'hui à montrer comment ces espaces peuvent constituer des environnements potentiellement fertiles pour les activités productives et marchandes. Ce paradigme s'est substitué au point de vue qui considérait que la partition coloniale n'avait été motivée que par des considérations géopolitiques, ignorantes du désir des ethnies à vivre dans des territoires homogènes du point de vue culturel (voir IGUÉ 1989). Les géographes engagés dans la réflexion sur les frontières ouest-africaines en sont venus à considérer ces espaces comme une partie centrale de réseaux économiques fondés sur un certain nombre de centres urbains
(Club du Sahel 2003; Codo 1986; GréGoire \& LabaZÉE 1993). Généralement, ces réseaux sont hiérarchisés, c'est-à-dire que les flux marchands s'organisent dans un fuseau urbain transfrontalier coupé par une frontière administrative considérée comme un accélérateur des dynamiques commerciales. Les réseaux sont alors basés sur l'exploitation des ressources agricoles, la possibilité de bénéficier de positions stratégiques du point de vue des flux d'import-export ainsi que sur les capacités des marchands locaux.

Cette représentation permet de montrer que les petites et moyennes villes fonctionnent comme des relais en direction des centres de consommation et de décision situés en périphérie du système (COUR \& SNRECH 1998). Il n'existe pour ainsi dire pas réellement de «région» frontalière, car les flux qui transitent par la frontière sont organisés dans une perspective qui prend pour cadre les opportunités économiques à l'échelle internationale. Cette conception s'accommode également des potentialités productives locales car elle permet à une région de bâtir une économie très largement extravertie en direction des grands marchés régionaux. Elle présente alors les frontières comme des espaces générateurs d'opportunités économiques.

Dans ce modèle, les acteurs doivent une grande partie de leurs profits à l'existence même des frontières nationales, instaurant des variations tarifaires et législatives dont ils usent et parfois abusent (Boluvi 2004). C'est le cas des patrons qui constituent des sociabilités particulières basées sur des réseaux de parenté et sur des alliés choisis en fonction de leur influence (WALTHER 2005). Le modèle est également ouvert aux stratégies de branchements des acteurs formels comme les douaniers ou les policiers, qui agissent dans le cadre de l'Etat-nation et qui régulent, par leur présence ou leur absence, les flux traversant la frontière. Loin de constituer des zones d'exception à l'échelle nationale, les espaces frontaliers forment les postes avancés des économies nationales dans lesquels les élites des plus grands centres participent au commerce (BACH 1998). Plus que jamais, dans un contexte de crise des économies de production, les Etats vivent de leurs frontières, dont l'organisation locale et régionale s'inscrit en continuité de l'organisation générale de l'espace socioéconomique.

\section{Gaya-Malanville-Kamba: un marché régional à la centralité mouvante}

Le réseau urbain dans lequel s'inscrivent les dynamiques commerciales de Gaya-Malanville-Kamba ne constitue pas un cas unique à l'échelle de l'Afrique sahélienne. Du sud du Sénégal au nord du Cameroun, une dizaine de réseaux dessinent une large bande 
zonale encore discontinue, à l'intérieur de laquelle quatre d'entre eux intéressent particulièrement la frontière entre Niger, Bénin et Nigeria: Zinder-Kano, Maradi-Katsina, Birni N'Konni-Illéla- Sokoto et GayaMalanville-Kamba (carte 1).

En considérant uniquement la population urbaine comme facteur de centralité, le sommet de la hiérarchie régionale du réseau qui fait l'objet de l'étude est occupé par les villes de Niamey et Sokoto, les centres de rang intermédiaire sont formés de Parakou, Kandi, Birni N'Kebbi, Jega, Illéla et Gunmi alors les centres de Gaya (33'000 hab.), Malanville (58'000 hab.) et Kamba (30'000 hab.) appartiennent à l'échelon inférieur, comme une quinzaine d'autres cités nigériennes, béninoises et nigérianes (carte 2). L'ensemble des centres concernés est structuré selon un modèle tripolaire dans lequel les villes de Gaya, Malanville et Kamba occupent le coeur d'un fuseau qui relie Niamey, Cotonou par l'intermédiaire de Parakou, et Lagos par le biais des grandes villes de l'ouest du Nigeria. Cependant, cette image fonctionnelle ne révèle qu'une part superficielle de l'organisation de l'espace économique sahélien, caractérisé par un degré élevé d'informalité.

En effet, le succès d'un marché est très largement dû à la libre circulation des produits à travers les frontières nationales et, consécutivement, à la possibilité pour les acheteurs des trois Etats de franchir ces frontières à un coût minimal. Dans cette organisation, le rôle du Nigeria est prépondérant, compte tenu d'une part de l'importance de son marché intérieur qui représente $60 \%$ des consommateurs et $50 \%$ du potentiel industriel de l'Afrique de l'Ouest (Programme des Nations Unies POUR LE DÉveloppement (PNUD) 2004), et d'autre part de sa politique douanière, caractérisée par des droits peu élevés sur les produits courants (lait) et des taux élevés pour les biens entrant en concurrence avec la production nationale (volaille, bière, maïs). Certains produits comme les pneumatiques, la friperie ou la farine de blé sont même frappés de prohibition.

Ces particularités favorisent une intense activité informelle. Des voies détournées sont alors utilisées pour acheminer les marchandises au Nigeria, moyennant certains accommodements avec les fonctionnaires chargés de la surveillance du territoire. Les produits qui font l'objet de ce commerce frauduleux suivent des itinéraires certes tortueux, mais bénéficient de complicités douanières évidentes et d'une coordination efficace. Le jeu des commerçants consiste alors à entretenir leurs relations auprès des douaniers autant qu'à prospecter de nouveaux itinéraires pour tenter de leur échapper temporairement. Ces stratégies ont des répercussions importantes sur la géographie marchande puisque, d'une manière assez systématique, les flux sont d'abord détournés de leur itinéraire officiel, avant d'être à nouveau restructurés selon des principes informels. Ce genre de démarche concerne tous les produits importés, notamment les biens industriels (friperie, cigarettes, hydrocarbures, véhicules d'occasion) et les denrées agricoles (riz, blé, maïs) qui transitent par Gaya et qui représentent une valeur déclarée de 9,29 mia de FCFA en 2005 (14,1 mio $€$ ).

\subsection{Gaya et Malanville: une convergence d'intérêts}

Le marché de Gaya présente une diversité de produits relativement importante dans un cadre urbain récemment rénové. Dans son enceinte, les activités commerciales obéissent à une organisation spatiale particulière dans laquelle 300 commerçants se regroupent par productions et par sexe: aux femmes la vente de fruits, de légumes, de poissons, de condiments, de grains au détail, de gari et d'igname; aux hommes celle de la viande, des plastiques, de l'alimentation générale, des textiles neufs et usagés, de la quincaillerie, des cosmétiques et des chaussures. Une importante superficie du marché est consacrée aux biens manufacturés d'origine chinoise ou nigériane tandis que les produits agricoles sont issus soit des jardins urbains et périurbains de Gaya, soit directement du Bénin voisin.

Malanville, elle, possède sans conteste le plus grand et le plus diversifié des marchés urbains de la zone frontalière. Il attire une clientèle internationale et fonctionne comme le lieu de concentration des produits agricoles locaux (riz, oignons) avant leur exportation vers le Niger ou le Nigeria. Son dynamisme provient essentiellement de sa situation géographique stratégique sur l'axe Cotonou - Niamey et d'une fiscalité modeste. C'est en effet à Malanville que la proportion de marchands d'origine allogène est la plus forte $(90 \%)$ alors que, de ce point de vue, Gaya et Kamba possèdent un profil plus marqué par des commerçants venant de la ville même, de l'Etat ou du Département proches. Ces caractéristiques s'expliquent par le fait que les populations locales (tchenga et songhay) ne présentaient pas d'héritages particuliers par rapport au commerce.

Entre Gaya et Malanville, les flux sont incessants, notamment parce que les consommateurs nigériens préfèrent s'approvisionner au Bénin plutôt que chez eux. L'argument le plus souvent évoqué pour justifier ce comportement est lié à la différence de prix qui avantagerait systématiquement Malanville au détriment de Gaya. Les données disponibles relatives aux variations mensuelles du prix des céréales entre les deux marchés de 2000 à 2005 montrent cependant que cet avantage n'est pas spectaculaire (fig. 1). D'une manière générale, les prix présentent de très fortes variations saisonnières pour tous les produits. La disponibilité variable des céréales en question engendre alors une forte augmentation conjoncturelle en période de 


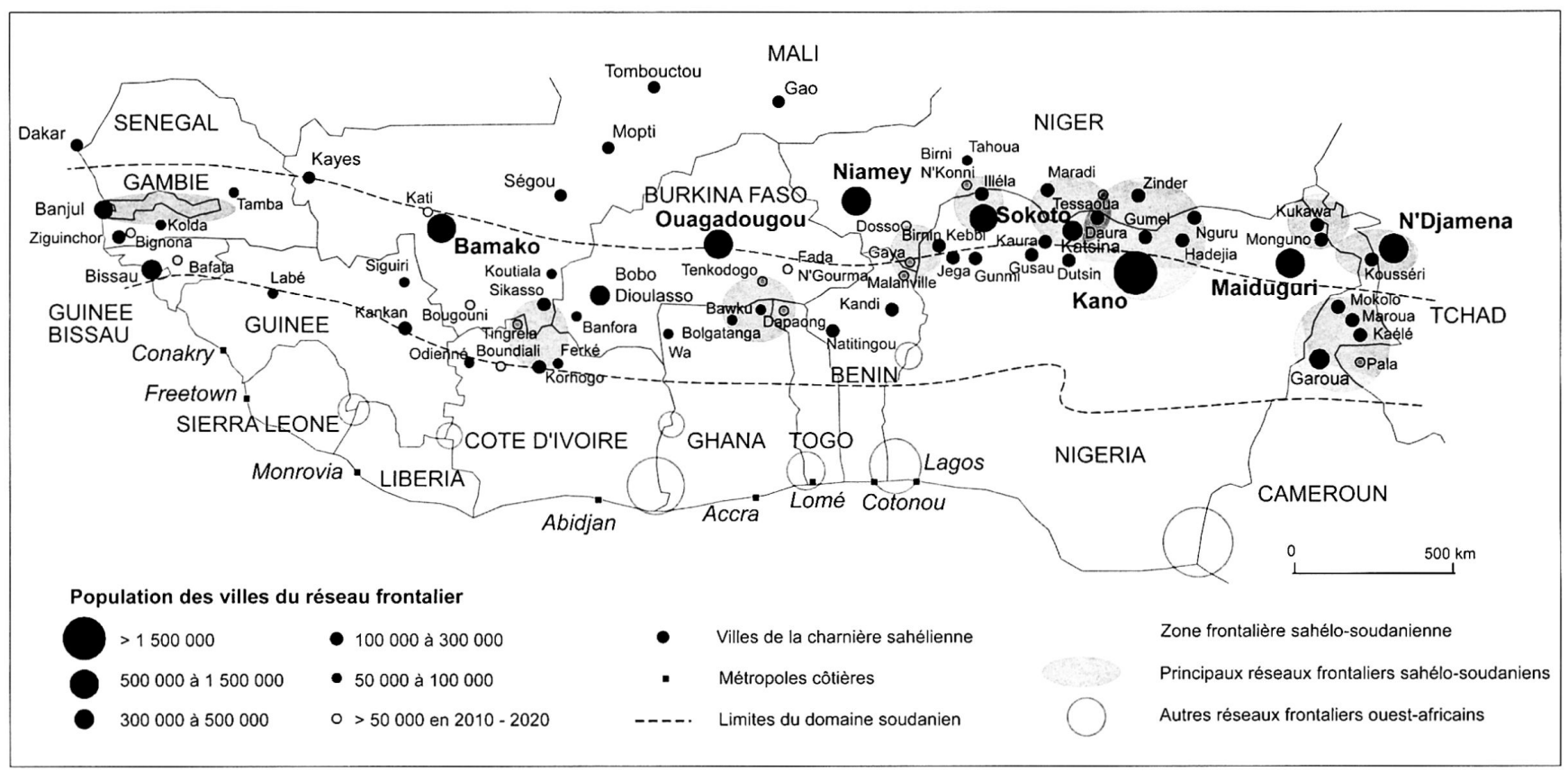

Carte 1: Réseaux transfrontaliers sahélo-soudaniens

Grenzüberschreitende Vernetzungen zwischen der Sahelzone und dem Sudan

Cross-border networks between the Sahel Zone and the Sudan

Source: Club du Sahel 2003; cartographie: Club du Sahel 2004, O. Walther 2005 


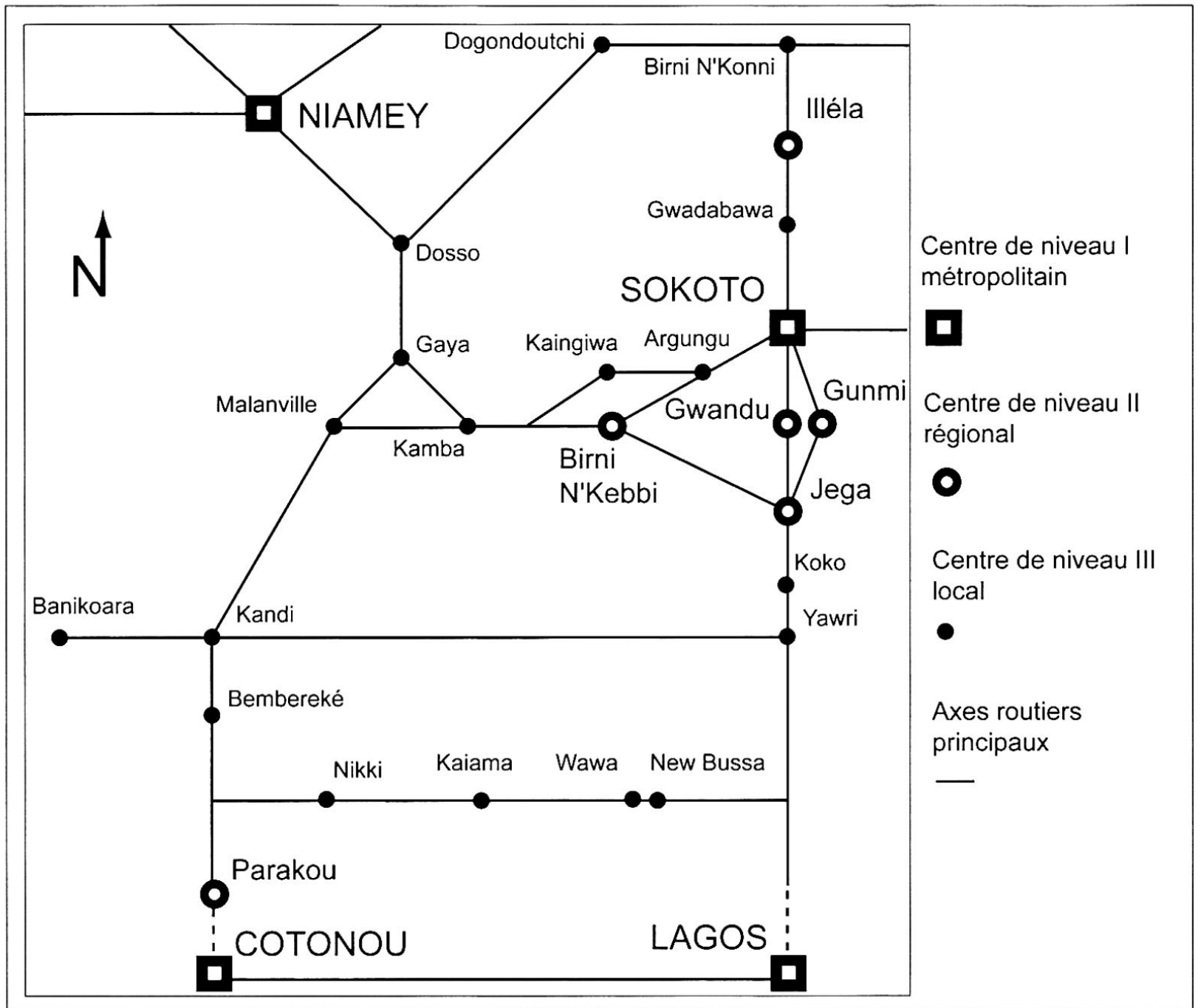

Carte 2: Hiérarchie urbaine de l'espace régional

Regionale Städtehierarchie

Regional urban hierarchies

Source: O. WALTHER 2005

pénurie (mai et juin) et, inversement, un effondrement des cours dans la période qui suit immédiatement les récoltes (septembre). De ce point de vue, les produits vendus dans les deux villes connaissent des variations annuelles très importantes liées à la disponibilité des stocks et, conjoncturellement, aux épisodes de famine qui frappent l'Afrique sahélienne.

Au cours de leurs variations saisonnières, les prix des céréales suivent cependant une évolution identique sur les deux marchés, autrement dit l'écart de prix est relativement constant. Certains écarts particulièrement marqués en défaveur de Gaya, comme en 2000, s'expliquent par des crises politiques qui entraînèrent la fermeture des frontières et l'impossibilité de ravitailler le Niger en céréales béninoises et non par un avantage concurrentiel déterminant du Bénin sur son voisin. De même, des problèmes frontaliers survenus au milieu de l'année 2001 entre les deux Etats ont entraîné un renchérissement du niébé nigérien au Bénin. Mais d'une manière générale, les prix des céréales montrent une tendance à l'égalisation de part et d'autre de la frontière depuis 2002. La différence est d'autant plus faible que les produits viennent du Niger: le mil est très légèrement plus cher à Gaya qu'à Malanville, le sorgho présente déjà des écarts plus prononcés en faveur du Bénin, davantage marqués pour le maïs. En définitive, les données disponibles tendent à montrer que le cours des céréales, quoique soumis à de fortes variations dues à des facteurs climatiques et politiques, connaît une convergence toujours accrue entre les deux marchés frontaliers. Par conséquent, le 


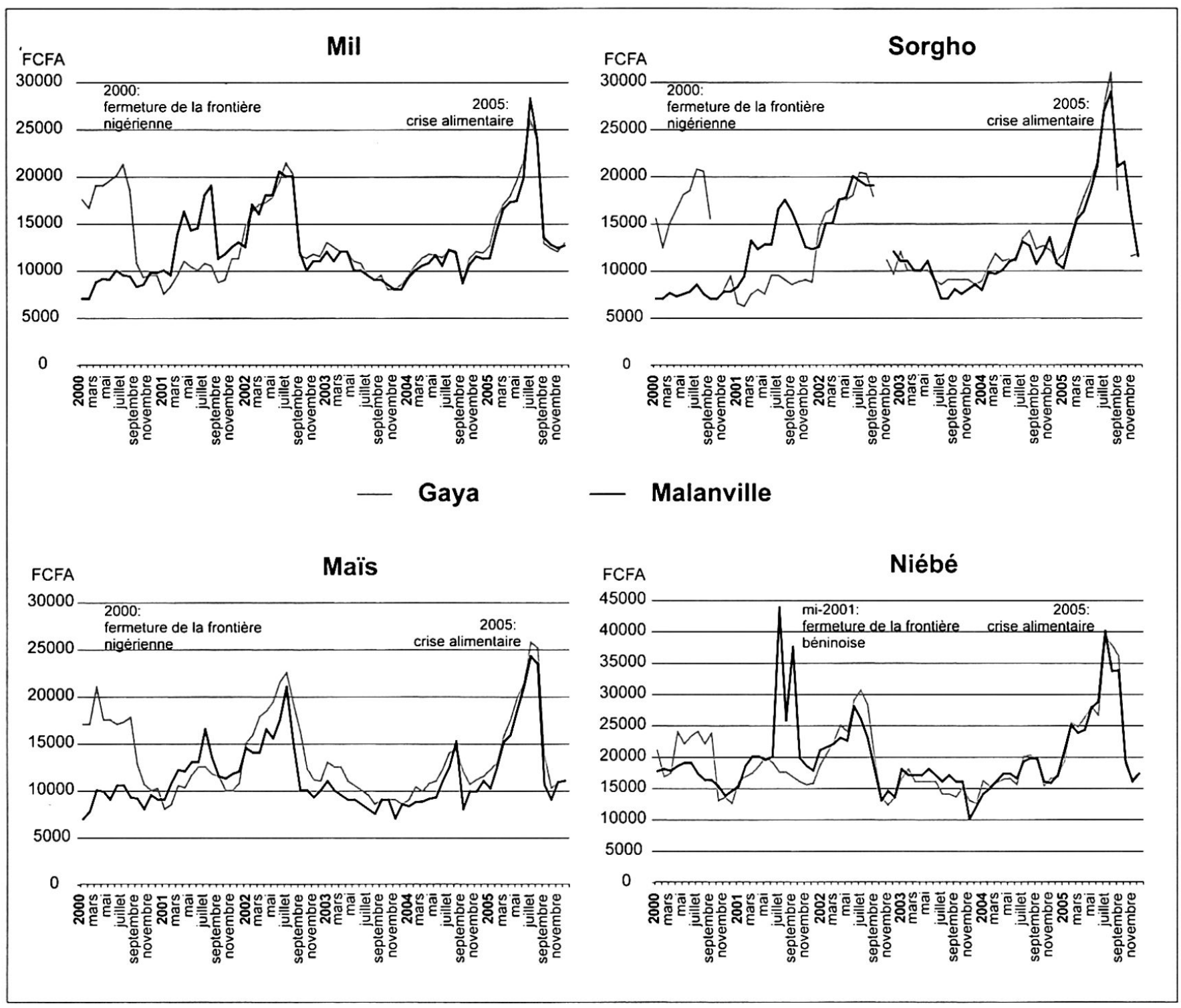

Fig. 1: Evolution du prix des céréales sur les marchés de Gaya (en gris) et de Malanville (en noir), 2000 à 2005 Entwicklung des Getreidepreises auf den Märkten von Gaya (grau) und Malanville (schwarz), 2000 bis 2005 Price trends of cereal on the markets of Gaya (grey line) and Malanville (black line) from 2000 to 2005 Source: Ministère du Développement communautaire, Direction régionale de Gaya 2005

gain espéré par le consommateur a significativement diminué depuis cinq ans.

En revanche, le passage des frontières constitue une opportunité toujours profitable pour les grossistes nigériens, qui déploient chaque semaine une intense activité économique entre les marchés de Gaya et de Malanville. Désireux de minimiser leurs frais de douane, ces commerçants acheminent leurs marchandises en charrettes depuis le Bénin jusqu'à Gaya tandis que des camions sont progressivement chargés de maïs, d'igname, de piment et de mil à destination des grands marchés de l'Ouest nigérien. D'autres commerçants préfèrent acheminer leurs céréales à Gaya par pirogue depuis le Bénin. Ils utilisent pour cela des petits ports «clandestins» qui permettent de limiter les frais de transport routier et de contourner les douanes et les syndicats frontaliers. Ce système assure des revenus à plusieurs dizaines de manœuvres et de conducteurs de véhicules et repose en grande partie sur le très faible coût de cette main-d'œuvre et la possibilité de bénéficier des réseaux de relations qui unissent les petits transporteurs et les agents de contrôle des frontières.

Si Malanville semble encore jouer un rôle régional plus développé que Gaya en ce qui concerne les 
céréales, l'évolution du commerce des vêtements d'occasion (friperie) montre que la rive nigérienne présente aujourd'hui un dynamisme plus prononcé qu'au Bénin. De 1989 à 1996, cette activité était pourtant bien développée dans la ville béninoise, grâce à des importations dirigées depuis les ports de Lomé et de Cotonou. Deux facteurs ont perturbé cette dynamique. D'une part, les clients qui venaient s'approvisionner au Bénin sont devenus eux-mêmes de grands opérateurs économiques préférant passer leurs commandes directement en Europe ou aux Etats-Unis, puis importer leurs vêtements en franchise de douanes jusqu'à Gaya. D'autre part, certaines pressions fiscales des autorités municipales de Malanville ont contribué au déménagement des grossistes en direction du Niger. L'interdiction d'importation du Nigeria et les profits qu'elle permet par contournements ont ensuite stimulé la concentration des activités entre les mains d'un petit nombre de grossistes à Gaya, tandis que les détaillants de Malanville perdaient une partie de leur meilleure clientèle.

En quinze ans, Gaya est alors devenue une des principales plaques tournantes de la friperie à l'échelle de l'Afrique de l'Ouest. Le choix de cette ville est éminemment stratégique car elle constitue le point le plus proche du port de Cotonou $(700 \mathrm{~km})$ d'où il est possible d'acheminer les textiles en franchise de douanes. Grâce à cette situation privilégiée, Gaya permet aux commerçants d'importer librement les textiles et de négocier leur vente à des marchands nigérians qui se chargeront de la réexportation dans leur pays et, dans une moindre mesure, vers le Tchad, le Cameroun, le Mali et le Burkina Faso. Ce commerce est dominé par une vingtaine de grands commerçants originaires du Niger, par des Igbo du Nigeria et par un petit nombre de marchands tunisiens et libanais, dont certains ont quitté Cotonou, Lomé ou le Burkina Faso pour s'implanter spécifiquement à Gaya. Cette activité représentait en 2005 plus de 8500 tonnes d'habits d'occasion pour un montant déclaré supérieur à 360 millions de FCFA $\left(549^{\prime} 000 €\right)$ et semble destinée à une croissance durable.

\subsection{Le déclin du marché de Kamba}

Dans l'espace frontalier, le marché de Kamba est sans conteste celui qui a le plus souffert d'une baisse de ses activités. Plus de $65 \%$ des commerçants enquêtés affirment que leurs affaires ont décliné en 2005 alors que cette proportion n'est que de $20 \%$ à Malanville et de $10 \%$ à Gaya. Jusqu'à la fin des années 1980 , Kamba était pourtant réputé à l'échelle internationale: les citadins de Niamey et de Gaya s'y approvisionnaient en produits agricoles, en carburant et en biens manufacturés. Ce temps est aujourd'hui révolu et le marché de Kamba semble progressivement vidé de ses marchands comme de ses chalands. Le déclin de la ville a des origines diverses, liées à l'évolution du contexte politique, économique et religieux du Nigeria.

La chute des activités urbaines s'explique tout d'abord par les limitations douanières du Nigeria vis-à-vis de ses voisins. La mise en place de ces mesures, conjuguée à des saisies de douanes et à un renforcement des contrôles a contribué au déclin du commerce. A cela s'ajoute l'insécurité dans les déplacements des marchands, liée à l'incapacité du gouvernement fédéral à assurer la surveillance de son territoire et aux nombreuses attaques à main armée. L'insécurité et la corruption ont donc une influence directe sur la fréquentation des routes et leur état et limitent considérablement les échanges économiques avec Gaya et Malanville.

Le dynamisme de la région est également très fortement influencé par la situation économique au Nigeria, elle-même liée aux pénuries structurelles, aux sabotages des oléoducs et raffineries et à l'évolution des cours du pétrole sur le marché mondial (Daloz 2002). Ce dernier facteur a été particulièrement déterminant dans la chute des activités de Kamba. En effet, l'attractivité de la ville a singulièrement diminué depuis que le gouvernement nigérian s'est donné pour but de relever les tarifs des produits pétroliers, une manière de ne plus subventionner massivement les prix à la consommation et, peut-être, de réduire la contrebande. Ainsi, malgré une hausse annuelle des prix à la consommation de $28 \%$ (Programme des Nations Unies pour le DéveLOPPEMENT (PNUD) 2004), le litre d'essence est passé de 0,7 à 30 nairas entre 1991 et 2000 (Soulé 2000). Le relèvement progressif des tarifs officiels n'a pas éliminé totalement le marché noir, qui assure entre 35 et $50 \%$ des besoins du Niger (Soulé 2000). En conséquence, la contrebande reste vive dans l'ensemble de l'espace frontalier. La lutte contre ce phénomène manque de moyens et de volonté politique dans la mesure où peu d'élus sont prêts à prendre des mesures coercitives à l'encontre des marchands illégaux qui réalisent encore des bénéfices sur les produits pétroliers.

Enfin, du point de vue religieux, l'exacerbation des conflits entre chrétiens et musulmans, associée à l'application de la charia dans les Etats du nord du Nigeria a conduit à des heurts défavorables pour l'activité économique de Kamba. Igbo et Yoruba chrétiens, progressivement installés depuis les années 1930, ont alors déserté la ville pour Ouagadougou, Niamey, Gaya et Malanville. Ces départs ont encore fragilisé l'économie de Kamba, à l'avantage des deux autres centres frontaliers. Aujourd'hui, les Igbo et les Yoruba ne représentent plus que 20 et $10 \%$ des commerçants enquêtés, une proportion supérieure à celle de Malanville et Gaya où ces deux groupes forment ensemble 5 à $10 \%$ des marchands. Leur départ a précipité le déclin 
économique de la ville dans la mesure où il s'agissait de marchands spécialisés dans la vente de produits manufacturés, notamment les plastiques, les machines, la quincaillerie et les pièces détachées en provenance des métropoles industrielles du sud du Nigeria (Lagos, Onitscha, Ibadan). En quittant la ville, leur savoir-faire en matière de réseaux d'approvisionnement n'a pas été remplacé par des compétences locales.

\section{Conclusion: centralité mobile et programmes transfrontaliers de développement}

Dans l'espace frontalier de Gaya-Malanville-Kamba, les relations entretenues par les marchés urbains oscillent entre concurrence et complémentarité. Du fait d'une décentralisation insuffisamment capable de renforcer le rôle des pouvoirs publics dans la gestion territoriale, une grande liberté d'action est laissée aux agents économiques privés. Ces patrons développent des affinités transfrontalières mouvantes, dont l'intensité est très largement dépendante de stratégies informelles. L'exemple des flux de céréales qui transitent entre Gaya et Malanville montre qu'une certaine convergence est à l'œuvre entre les deux villes. Celle-ci s'explique par le degré d'ouverture des frontières et les nombreuses stratégies mises en place par les opérateurs pour faciliter l'approvisionnement de l'une ou de l'autre ville. Elle illustre la mise en place d'un «marché commun» plus équilibré que par le passé mais encore fortement dépendant des crises politiques qui provoquent de brusques augmentations des cours. L'évolution du commerce de la friperie présente une évolution différente, marquée par la prépondérance de Gaya sur Malanville, grâce à un environnement fiscal plus attrayant, permettant à la ville nigérienne de devenir un centre d'envergure internationale au détriment de sa voisine.

En ce qui concerne les relations entretenues avec la ville nigériane de Kamba, force est de constater qu'un ensemble de facteurs politiques, économiques et religieux a conduit au dépérissement du marché au profit de Gaya et de Malanville. D'une part, les politiques restrictives en matière d'importation, associées à une tolérance douanière plus faible, ont conduit un nombre croissant de Nigériens et de Béninois à s'approvisionner dans leur pays respectif. D'autre part, l'avantage comparatif dont bénéficiait Kamba en matière d'hydrocarbures s'est très largement réduit consécutivement à la hausse des prix à la consommation. Enfin, les violences religieuses qui se sont concentrées sur les chrétiens du nord du Nigeria ont entraîné le départ d'un nombre important de marchands spécialisés au profit des autres villes de la frontière. Ainsi, en même temps que la partie nigériane souffrait d'une érosion de ses avantages comparatifs, les petites villes nigérien- nes et béninoises développaient des relations toujours plus intenses, grâce à l'habileté et à la souplesse des patrons locaux. Il en résulte une forme de centralité tout à fait particulière: extrêmement mobile et sensible aux brusques fermetures des frontières, celle-ci se déplace au gré des affinités des agents économiques au niveau local et des opportunités économiques plus larges dues au contexte national et international.

Cette conclusion peut intéresser les politiques territoriales des organismes nationaux ou des agences de développement qui œuvrent dans la région. En effet, si les petites et moyennes villes frontalières renforcent leurs liens respectifs au travers d'une centralité mobile et partagent des préoccupations communes, cela signifie que les plans de développement conçus jusqu'ici sur le postulat d'une compétition à l'échelle nationale sont probablement dépassés. Peut-être est-ce le moment de repenser l'aide au développement local en appuyant des programmes transfrontaliers qui permettent à la fois de prendre en compte le potentiel important des agents économiques privés et la nécessité d'une régulation des flux marchands. En l'absence de politiques de développement traitant spécialement de la dimension circulatoire de l'économie, les patrons des régions frontalières demeurent des agents de leur propre économie et non des représentants des Etats ou d'entreprises ancrées dans des territoires de production et de commercialisation. L'observation des relations entretenues entre les marchés de Gaya, Malanville et Kamba montre donc à la fois les limites des contraintes pouvant être exercées par les trois Etats, mais également la nécessité du maintien de leur fonction de sécurité, à défaut de pouvoir intervenir avec efficacité dans le système économique frontalier.

\section{Remerciements}

L'auteur souhaite remercier le Réseau Universitaire International de Genève qui a financé une partie de ses travaux, au travers de son programme de recherche «Négocier les conflits d'intérêt liés à l'eau».

\section{Bibliographie}

BACH, D. (dir.) (1998): Régionalisation, mondialisation et fragmentation en Afrique subsaharienne. - Paris: Karthala.

Boluvi, G.-M. (2004): Malanville-Gaya: comptoir commercial et couloir de spéculations (pays-frontière de l'informel). - Paris: Club du Sahel et de l'Afrique de l'Ouest.

Club du SAHel (2003): Atelier régional sur la coopération transfrontalière en Afrique de l'Ouest. Frontières et intégration en Afrique de l'Ouest. - Paris: Club du Sahel - Organisation de coopération et de développement économiques (OCDE). 
Club du Sahel (2004): La coopération transfrontalière au service du processus d'intégration régionale. - Paris: Club du Sahel - OCDE.

Codo, L.C. (1986): Incidences économiques des flux frontaliers clandestins. Le cas du Nigeria et du Bénin. - In: Afrique contemporaine 140:11-23.

COUR, J.-M. \& S. SNRECH (dirs) (1998): Pour préparer l'avenir de l'Afrique de l'Ouest. Une vision à l'horizon 2020. Etudes des perspectives à long terme en Afrique de l'Ouest. - Paris: Club du Sahel - OCDE.

DALoz, J.-P. (2002): Elites et représentations politique. La culture de l'échange inégal au Nigeria. - Pessac: Presses Universitaires de Bordeaux.

Direction Générale des Douanes (2005): Exportation par le bureau de Gaya. - Niamey: République du Niger, Direction des Recettes et des Statistiques.

Grégorre, E. \& P. Labazée (1993): Mobilité marchande et urbanisation. Le cas de Korhogo (Côted'Ivoire) et de Maradi (Niger). - In: Cahiers des Sciences Humaines 29, 2-3: 527-546.

IGUÉ, J.O. (1989): Le développement des périphéries nationales en Afrique. Tropiques. Lieux et liens. - Paris: Office de la recherche scientifique et technique outremer (ORSTOM).

Jaubert, R., Bakonyi-Moeschler, M., Caloz, R. \& J. Winistorfer (2005): L'eau: quelles crises dans les régions à fortes contraintes? - Genève: Réseau universitaire international de Genève (RUIG).

Programme des Nations-Unies pour le DéveloppeMENT (PNUD) (2004): Rapport mondial sur le développement humain. - Paris: Economica.

ReTAillé, D. (1989): Comment lire le contact SaharaSahel? In: De l'Atlantique à l'Ennedi. - Abidjan: Centre Culturel Français: 17-33.

Retaillé, D. (2005): L'espace mobile. - In: Antheaume, B. \& F. Giraut (éds): Le territoire est mort. Vive les territoires! Une (re)fabrication au nom du développement. - Paris: Institut de recherche pour le développement (IRD): 175-202.

SoulÉ, B.G. (2000): Les perspectives des échanges entre le Nigeria et ses voisins. - Paris: Club du Sahel - OCDE.

Satterthwaite, D. \& C. Tacoli (2003): The urban part of rural development. The role of small and intermediate urban centres in rural and regional development and poverty reduction. - London: International Institute for Environment and Development (IIED).

Walther, O. (2004): Au-delà de l'opposition entre villes et campagnes. Eléments pour un modèle territorial dynamique en Afrique de l'Ouest. - In: L'Information géographique 68: 308-319.

WalTheR, O. (2005): «Merci patron...». L'espérance de recevoir et l'obligation d'offrir dans les relations clientélistes au Niger et au Bénin. - In: Dambo, L. \& E. REYNARD (éds): Vivre dans les milieux fragiles. Alpes et Sahel. - Lausanne: Travaux et Recherches de l'Institut de Géographie de l'Université de Lausanne 31:309-325.

\section{Résumé: Villes-frontières et réseaux informels sahéliens (Gaya-Malanville-Kamba)}

Cet article traite des relations économiques entretenues par trois villes-marchés situées sur la frontière entre le Niger, le Bénin et le Nigeria. En focalisant l'intérêt sur les liens qui unissent les commerçants des marchés locaux - appelés patrons - il montre l'extrême souplesse qui caractérise leurs stratégies et le caractère profondément informel de leurs activités. $\mathrm{Au}$ travers des exemples du commerce des produits de rente, des flux d'import-export et des activités de détail, il analyse les changements survenus dans la centralité des villes de Gaya, Malanville et Kamba. Ce faisant, il s'attache à montrer de quelle manière les villes en question forment un espace économique frontalier basé sur des rapports de concurrence et de complémentarité. Cette particularité permet de conclure en faveur d'un renouvellement des approches de développement local, qui prenne en considération le potentiel important des agents économiques privés et la nécessité d'une régulation des flux marchands, par l'intermédiaire de politiques publiques spécifiquement adaptées aux problèmes que rencontrent ces espaces.

\section{Abstract: Cross-border cities and informal networks in the Sahel (Gaya-Malanville-Kamba)}

This article deals with the economic relationships between three market cities located on the border of Niger, Benin and Nigeria. Focusing on the links that unite the businessmen of the local markets - the socalled patrons - it reveals the extreme flexibility of their strategies as well as the deeply informal nature of their activities. Through the analysis of examples taken from the commerce of agricultural products, import and export flows and detail activities, it studies the changes that have taken place in the city centres of Gaya, Malanville and Kamba. Thus, the article is able to highlight how these cities have developed a crossborder economic area on the basis of competitive and complementary economies. It suggests the need for new local development approaches, which take the important potential of private economical actors into account. Further, it is argued that commercial flows may also be regulated with the help of public policies, as long as they are specifically adapted to the problems that these areas face.

\section{Zusammenfassung: Grenzstädte und informelle Vernetzungen im Sahel (Gaya-Malanville-Kamba)}

Dieser Artikel beschreibt die wirtschaftlichen Verflechtungen dreier afrikanischer Märkte im Grenzdreieck von Niger, Benin und Nigeria. Dabei gilt das Hauptaugenmerk den gemeinsamen Interessen der Händler aller drei Standorte. Es wird auf die aussergewöhnliche Flexibilität der Marktstrategien und das überaus informelle Geschäftsgebaren eingegangen. Eine Auflistung von Beispielen der Landwirtschafts- 
produkte, der Import- und Exportbewegungen und der Einzelhandelsaktivitäten erlaubt die Analyse der Veränderungen der Städte Gaya, Malanville und Kamba im Hinblick auf ihre jeweilige Marktstellung. Dadurch wird aufgezeigt, auf welche Weise die untersuchten Städte auf der Grundlage von Konkurrenz und Komplementärwirtschaft einen grenzüberschreitenden Wirtschaftsraum bilden. Diese hier nachgewiesenen besonderen Rahmenbedingungen sprechen für eine Erneuerung lokaler Entwicklungsmassnahmen. Dabei erfordern das bedeutende Potential privater Wirtschaftsfaktoren und die Notwendigkeit einer Regulierung der Warenströme die Einschaltung einer an die Besonderheiten dieses Wirtschaftsraumes angepassten öffentlichen Hand.

Dr. Olivier Walther, Département Géographie et Développement (GEODE), Centre de Populations, de Pauvreté et de Politiques Socio-Economiques (CEPS/ INSTEAD), BP 48, L-4501 Differdange, Luxembourg. e-mail: Olivier.Walther@ceps.lu

Manuskripteingang/received/manuscrit entré le 11.7.2006

Annahme zum Druck/accepted for publication/accepté pour l'impression: 25.1.2007 